\title{
Interrogating the Temporal Coherence of EUV Frequency Combs with Highly Charged Ions
}

\author{
Chunhai Lyu $\odot,{ }^{*}$ Stefano M. Cavaletto $\odot,{ }^{\dagger}$ Christoph H. Keitel $\odot$, and Zoltán Harman \\ Max-Planck-Institut für Kernphysik, Saupfercheckweg 1, 69117 Heidelberg, Germany
}

(Received 6 June 2020; accepted 7 August 2020; published 25 August 2020)

\begin{abstract}
A scheme to infer the temporal coherence of EUV frequency combs generated from intracavity highorder harmonic generation is put forward. The excitation dynamics of highly charged Mg-like ions, which interact with EUV pulse trains featuring different carrier-envelope-phase fluctuations, are simulated. While demonstrating the microscopic origin of the macroscopic equivalence between excitations induced by pulse trains and continuous-wave lasers, we show that the coherence time of the pulse train can be determined from the spectrum of the excitations. The scheme will provide a verification of the comb temporal coherence at timescales several orders of magnitude longer than current state of the art, and at the same time will enable high-precision spectroscopy of EUV transitions with a relative accuracy up to $\delta \omega / \omega \sim 10^{-17}$.
\end{abstract}

DOI: 10.1103/PhysRevLett.125.093201

A train of evenly delayed coherent electromagnetic pulses resembles a structure in the frequency domain with uniformly displaced frequency peaks, i.e., a frequency comb (FC) [1,2]. The inverse of the coherence time $\tau_{c}$ of such a FC determines the width of each comb tooth, and can be inferred by measuring the beating spectra between the corresponding pulse train and an independent ultrastable continuous-wave (cw) reference laser [3,4]. For optical FCs, coherence times longer than $1 \mathrm{~s}$, or tooth widths narrower than $1 \mathrm{~Hz}$, have been measured [3,4], which allows wide applications of FCs in high-precision spectroscopy [5,6], the search for exoplanets $[7,8]$ and the construction of ultrastable optical atomic clocks [9].

Through intracavity high-order harmonic generation (HHG) [10] of femtosecond infrared (IR) pulse trains, coherent extreme-ultraviolet (EUV) pulse trains representing EUV FCs have been demonstrated [11,12]. This could allow for high-precision spectroscopy in the EUV regime $[13,14]$ and enable next-generation atomic clocks based on EUV transitions [15-17]. However, due to the lack of cw EUV reference lasers, the temporal coherence of an EUV FC is mainly investigated by splitting the EUV pulse train into two pathways and then recombing them to perform Michelson interference [12,18]. The observed cross correlation between two adjacent pulses reveals the well-defined temporal coherence on the time scale of $10 \mathrm{~ns}$ [18]. This result was further verified by the direct

Published by the American Physical Society under the terms of the Creative Commons Attribution 4.0 International license. Further distribution of this work must maintain attribution to the author(s) and the published article's title, journal citation, and DOI. Open access publication funded by the Max Planck Society. frequency-comb spectroscopy (DFCS) of atomic transitions in $\mathrm{Ne}$ and $\operatorname{Ar}$ [13]: the measured fluorescence spectra exhibited a full-width at half-maximum (FWHM) of $10 \mathrm{MHz}$, implying that the coherence time of the EUV $\mathrm{FC}$ is longer than $16 \mathrm{~ns}$.

Instead of splitting the EUV pulse train, the IR pulse train can be split and sent into two isolated cavities where HHG takes place separately [19]. The cross-correlation measurement of the two almost independently generated EUV pulse trains indicates that HHG itself may be the leading process affecting the coherence time of the EUV frequency combs [19]. This suggests that when the IR frequency comb is locked to a $\mathrm{mHz}$ ultrastable $\mathrm{cw}$ laser $[3,20]$, EUV FCs with coherence times of $\tau_{c} \gtrsim 1 \mathrm{~s}$ (tooth width $\lesssim 160 \mathrm{mHz}$ ) could be obtained. However, recent studies [21,22] argue that such a fine comb structure may not be achieved with currently available feedback loops, and have set an ultimate upper limit on the comb coherence time of EUV FCs of $\tau_{c} \lesssim 64 \mathrm{~ns}$ (tooth width $\gtrsim 2.5 \mathrm{MHz}$ ). Therefore, verifying the coherence time of the EUV frequency comb at longer timescales becomes essential. Currently, this is limited either by the longest arm length tunable in the Michelson-interference schemes $[12,18]$ or by the longest lifetimes of the EUV transitions available in the DFCS schemes [13].

Highly charged ions (HCIs) can be produced, e.g., in an electron-beam ion trap (EBIT) [23] and then be moved to a cryogenic Paul trap [24-26] for interactions with external lasers [26-28]. Due to the existence of environmentinsensitive forbidden optical transitions, HCIs are of great interest in frequency metrology and for tests of fundamental physics $[29,30]$ such as testing the hypothesis of fine-structure-constant variation [31-34] and for constraining the strength of a possible fifth force acting 
TABLE I. Transition energy $\hbar \omega$ and lifetime $\tau$ of the $[\mathrm{Ne}] 3 s 3 p$ states in Mg-like ions. The lifetimes for $\mathrm{Ca}^{8+}$ and $\mathrm{Ti}^{10+}$ are adopted from Ref. [37].

\begin{tabular}{|c|c|c|c|c|c|c|c|}
\hline \multirow[b]{2}{*}{ Ions } & \multicolumn{2}{|c|}{${ }^{1} P_{1}$} & \multicolumn{2}{|c|}{${ }^{3} P_{2}$} & \multicolumn{2}{|c|}{${ }^{3} P_{1}$} & \multirow{2}{*}{$\frac{{ }^{3} P_{0}}{\hbar \omega(\mathrm{eV})}$} \\
\hline & $\hbar \omega(\mathrm{eV})$ & $\tau$ & $\hbar \omega(\mathrm{eV})$ & $\tau$ & $\hbar \omega(\mathrm{eV})$ & $\tau$ & \\
\hline $\mathrm{S}^{4+}$ & 15.765 & $301 \mathrm{ps}$ & 10.434 & $16 \mathrm{~s}$ & 10.339 & $6.7 \mu \mathrm{s}$ & 10.294 \\
\hline $\mathrm{Ar}^{6+}$ & 21.167 & $123 \mathrm{ps}$ & 14.331 & $5.3 \mathrm{~s}$ & 14.122 & $1.3 \mu \mathrm{s}$ & 14.023 \\
\hline $\mathrm{Ca}^{8+}$ & 26.592 & $94 \mathrm{ps}$ & 18.339 & $2.2 \mathrm{~s}$ & 17.937 & $0.4 \mu \mathrm{s}$ & 17.752 \\
\hline $\mathrm{Ti}^{10+}$ & 32.108 & $72 \mathrm{ps}$ & 22.487 & $0.4 \mathrm{~s}$ & 21.790 & $0.1 \mu \mathrm{s}$ & 21.476 \\
\hline
\end{tabular}

between electrons and nucleons [35,36]. By employing excited configurations, specific HCIs also provide forbidden transitions that can be probed by EUV frequency combs. This would enable the detection of the coherence time of EUV pulse trains at timescales longer than $100 \mathrm{~ns}$, and at the same time render high-precision spectroscopy of EUV transitions possible.

In this Letter, we put forward the interrogation of the coherence time of an EUV FC with highly charged Mg-like ions featuring a ground-state configuration of $[\mathrm{Ne}] 3 s^{2}{ }^{1} S_{0}$. The energies and lifetimes of the $[\mathrm{Ne}] 3 s 3 p$ excited-state configurations for selected ions are presented in Table I. These values are calculated employing multiconfiguration Dirac-Hartree-Fock (MCDHF) theory [37,38] and referenced with the experimental values available from the NIST atomic database [39]. In contrast to the EUV transitions in neutral atoms that usually decay within 100 ns [13,14,40-44], the EUV transitions in Table I possess lifetimes around both $1 \mu \mathrm{s}$ and $1 \mathrm{~s}$. Therefore, they can be used to investigate the coherence time of an EUV pulse train for harmonics from the 9th to the 19th order and beyond. By extending the light-matter interaction to account for phase fluctuations in the pulse train, we show that the coherence time can be determined either through DFCS, where millions of pulses interact with the ion $[13,45]$, or via Ramsey frequency comb spectroscopy (RFCS) [14,40,41,43,44,46], where only two pulses separated in time interact with the ion.

$\mathrm{Mg}$-like $\mathrm{Ar}^{6+}$.- We consider $\mathrm{Mg}$-like $\mathrm{Ar}^{6+}$ ions as shown in Fig. 1: the ${ }^{1} P_{1}$ state decays to the ground state through a fast $E 1$ transition within one cycle of the EUV pulse, while the ${ }^{3} P_{1}$ and ${ }^{3} P_{2}$ states can effectively interact with hundreds and millions of pulses before they decay, and thus interrogate the temporal coherence of the EUV pulse trains at time scales around $1.3 \mu \mathrm{s}$ and $5.6 \mathrm{~s}$, respectively. State-of-the-art experimental energies [47] of the transitions from the ${ }^{3} P_{1}$ and ${ }^{3} P_{2}$ states to the ground state are $14.12248(24)$ and $14.33133(25) \mathrm{eV}$, respectively, with a relative uncertainty of $\delta \omega / \omega \sim 1.7 \times 10^{-5}$. We will show that the investigations of the FC coherence time can lead to a reduction of this uncertainty by several orders of magnitude.

Frequency comb.-In the time domain, a FC is described as a train of consecutive pulses with a repetition time of $T_{r}$ $[1,2]$ :

$$
E(t)=E_{p} \sum_{j} f\left(t-j T_{r}\right) \cos \left[\omega_{0} t+\phi(t)\right],
$$

where $E_{p}$ is the peak strength of the electric field, $f\left(t-j T_{r}\right)$ is the normalized envelope of the $j$ th pulse under a carrier frequency of $\omega_{0}$, and $\phi(t)$ is the phase noise at time $t$. For an ideal case where all these parameters are stable and deterministic, one obtains an infinitely correlated pulse train with a perfect comb structure in the frequency domain. However, fluctuations in $T_{r}$ and $\phi(t)$ lead to a finite correlation time that broadens the line shape of each tooth $[21,22,48-50]$. Here, we only consider the phase fluctuations on top of the carrier-envelope phase (CEP), which we model as a random walk process such that $[21,22]$

$$
\phi(t)=\int_{0}^{t} s\left(t^{\prime}\right) d t^{\prime}
$$

where $s(t)$ represents a Gaussian white noise with autocorrelation $\left\langle s(t) s\left(t^{\prime}\right)\right\rangle=\sigma^{2} \delta\left(t-t^{\prime}\right)$. This results in a coherence time of $\tau_{c}=1 / 2 \pi \sigma^{2}$, corresponding to a tooth FWHM of $\sigma^{2}$ [22].

Bloch equations.-We provide quantum dynamical simulations of the excitations of $\mathrm{Ar}^{6+}$ ions coupled to an EUV pulse train. The duration of each pulse is assumed to be 200 fs with a repetition time of $T_{r}=10 \mathrm{~ns}$ [27], corresponding to a FWHM bandwidth of $2.19 \mathrm{THz}$ and repetition rate of $100 \mathrm{MHz}$. The carrier frequency $\omega_{0}$ is

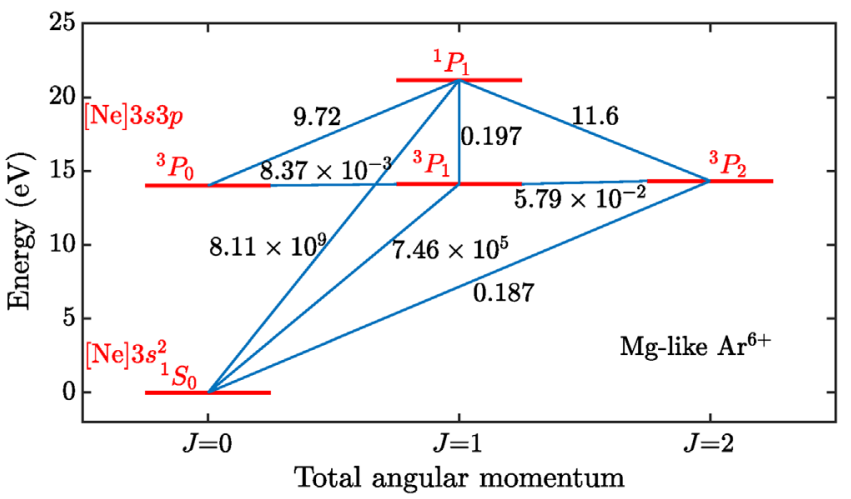

FIG. 1. Level structure and radiative transition rates (in units of per second) of $\mathrm{Ar}^{6+}$. 
tuned to the ${ }^{3} P_{1} \rightarrow{ }^{1} S_{0}$ transition. The energy separations between the levels shown in Table I are much larger than the bandwidth of the frequency comb. Therefore, the ions can be modeled as two-level systems whose dynamics are described by Bloch equations [51-54] in the rotating-wave approximation:

$$
\begin{aligned}
& \dot{\rho}_{\mathrm{ee}}=-\operatorname{Im}\left[\mu^{*} \mathcal{E}^{*}(t) \rho_{\mathrm{eg}}(t)\right]-\Gamma \rho_{\mathrm{ee}}(t), \\
& \dot{\rho}_{\mathrm{gg}}=\operatorname{Im}\left[\mu^{*} \mathcal{E}^{*}(t) \rho_{\mathrm{eg}}(t)\right]+\Gamma \rho_{\mathrm{ee}}(t), \\
& \dot{\rho}_{\mathrm{eg}}=\frac{i \mu \mathcal{E}(t)}{2}\left[\rho_{\mathrm{ee}}(t)-\rho_{\mathrm{gg}}(t)\right]+\left(i \Delta-\frac{\Gamma}{2}\right) \rho_{\mathrm{eg}}(t) .
\end{aligned}
$$

Here, $\rho_{\mathrm{ee}}(t)$ and $\rho_{\mathrm{gg}}(t)$ are the populations of the excited and ground states, respectively. $\rho_{\mathrm{eg}}(t)=\rho_{\mathrm{ge}}^{*}(t)$ is the offdiagonal element of the density matrix. $\mu$ is the dipole moment that couples to the field envelope

$$
\mathcal{E}(t)=E_{p} \sum_{j} f\left(t-j T_{r}\right) e^{i \phi(t)} .
$$

Furthermore, $\Gamma$ is the spontaneous-emission rate and $\Delta=\omega_{0}-\omega$ is the detuning.

Population dynamics. - Though most EUV FCs have an average power around tens of $\mu \mathrm{W}$ [10], an average power of several $\mathrm{mW}$ has been achieved recently [55]. Figure 2 shows two excitation dynamics induced by $4-\mathrm{mW}$ combs with different coherence times: while Figs. 2(a)-2(c) refer to a comb with $\tau_{c}=1 \mathrm{~s}$ as in Ref. [19], Figs. 2(d)-2(f) stand for the comb from Ref. [21] with $\tau_{c}=64 \mathrm{~ns}$. The EUV light is supposed to be focused onto a $10-\mu \mathrm{m}^{2}$ spot such that $E_{p}=1.19 \times 10^{8} \mathrm{~V} / \mathrm{m}$. The excitations (red lines) induced by a $170-\mathrm{nW}$ resonant $\mathrm{cw}$ laser, with the same fluctuating phase $\phi(t)$ but a constant field strength of $\mathcal{E}_{\text {eff }}=\int_{0}^{T_{r}} \mathcal{E}(t) d t / T_{r}=3580 \mathrm{~V} / \mathrm{m}$, are also shown. This cw laser, featuring a Rabi frequency of $720 \mathrm{kHz}$, bears the same power as the average power held by a single comb mode at $\omega_{0}$.

For both combs, one obtains coherent accumulations [56-59] of stepwise excitations [Figs. 2(a) and 2(d)]. The amount of each stepwise excitation within the 200-fs pulse duration is equivalent to the amount of continuous excitation by the corresponding $\mathrm{cw}$ laser within a period of $T_{r}=10 \mathrm{~ns}$, representing the microscopic origin of the macroscopic equivalence [45] between the pulse-train and cw-laser excitations illustrated in Figs. 2(b) and 2(e). While the similarities in the excitations by the first 15 pulses shown in Figs. 2(a) and 2(d) are clearly apparent for the two combs, differences start to emerge at times beyond the 64-ns-long coherence time of comb 2. For comb 1, whose CEP dephasing is negligible, the excitation by each pulse adds up coherently and induces the Rabi oscillation [60] shown in Figs. 2(a) and 2(b). For comb 2, however, the CEP dephasing starts to slow down the excitations, and a chaotic evolution is observed at long time scales [Figs. 2(d) and 2(e)].

Furthermore, when the time becomes much longer than the $1.3-\mu$ s excited-state lifetime, the coherent excitation of comb 1 evolves into a dynamical steady state [61-65]. The population decayed during the absence of the pulse within each cycle [blue line in Fig. 2(c)] is subsequently repumped by the next pulse, revealing the distinct transient behavior in comparison to the constant population (red line) induced
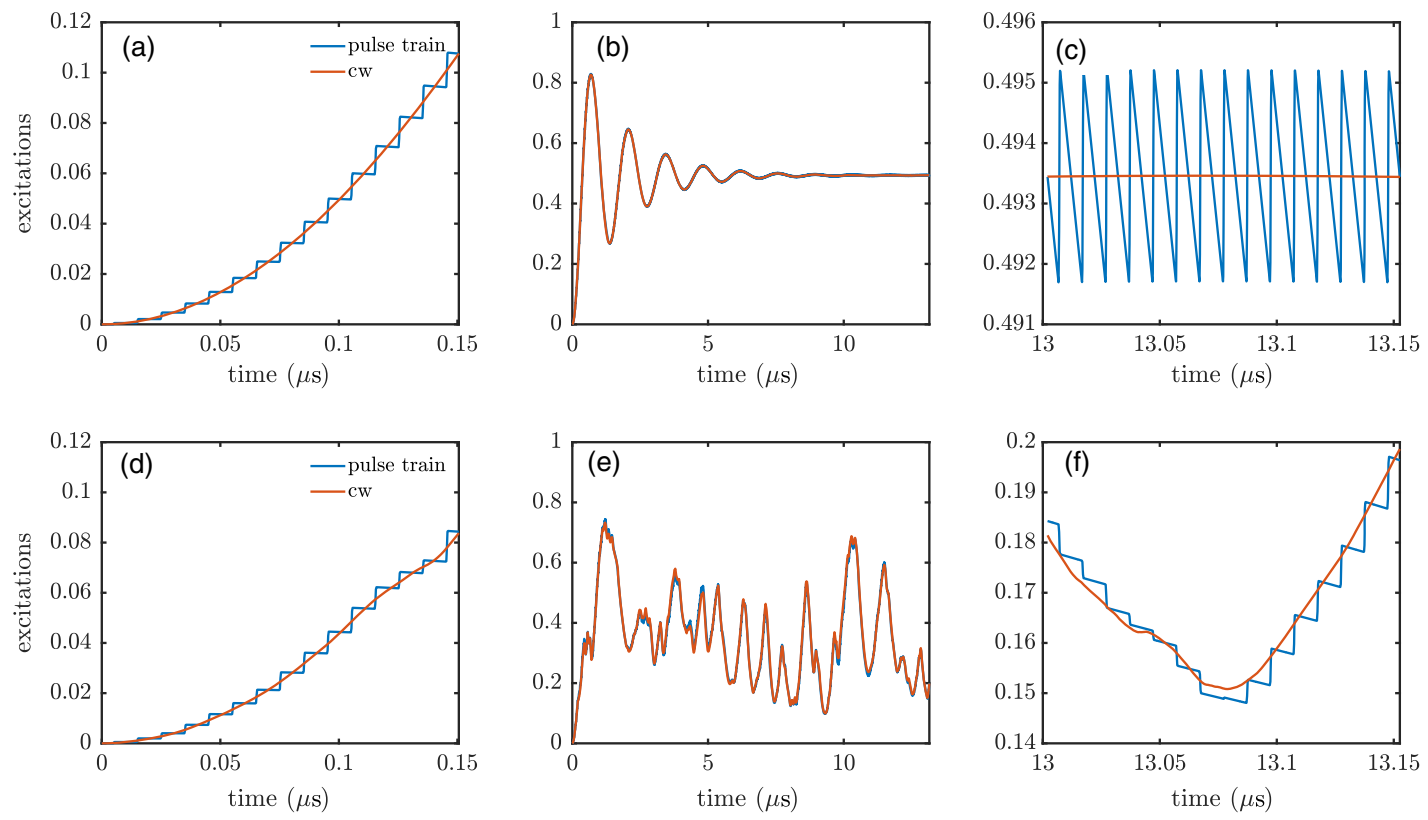

FIG. 2. The population $\rho_{\mathrm{ee}}(t)$ of the ${ }^{3} P_{1}$ state in $\mathrm{Ar}^{6+}$ induced by different 4-mW EUV FCs. (a)-(c) Comb 1 with $\tau_{c}=1 \mathrm{~s}$ [19]. (d)-(f) Comb 2 with $\tau_{c}=64$ ns [21]. Panels (a),(d) and (c),(f) show the excitations during the first and last 15 pulses in (b),(e), respectively. 
by cw lasers. The excitation dynamics of comb 2 , however, is always random and does not approach any steady state [Fig. 2(f)].

DFCS scheme.-To determine the coherence time of the FCs and the energy of the ionic transition, Fig. 3 illustrates the excitations as a function of the detuning $\Delta$. While Fig. 3(a) represents the steady-state excitation spectra for comb 1, the spectra in Fig. 3(b) for comb 2 are the average excitations over a duration of $1.3 \mathrm{~ms}$. The results for FCs with an average power of $40-\mu \mathrm{W}$ (blue lines) are also presented. For comb 1, whose $160-\mathrm{mHz}$ tooth width is much narrower than the $122-\mathrm{kHz}$ natural linewidth, the spectrum induced by a power of $40 \mu \mathrm{W}$ recovers the natural line shape of the corresponding ionic transition. The slightly broadened FWHM of $162 \mathrm{kHz}$ is a consequence of power broadening which becomes more significant at the power of $4 \mathrm{~mW}$ with a 8.5 -fold broadened FWHM of $1041 \mathrm{kHz}$. Nevertheless, measuring such spectra would enable the determination of the ${ }^{3} P_{1} \rightarrow{ }^{1} S_{0}$ transition energy in $\mathrm{Ar}^{6+}$ to a relative accuracy of $\delta \omega / \omega=10^{-11}$, with an improvement by more than 6 orders of magnitude compared to current results [47].
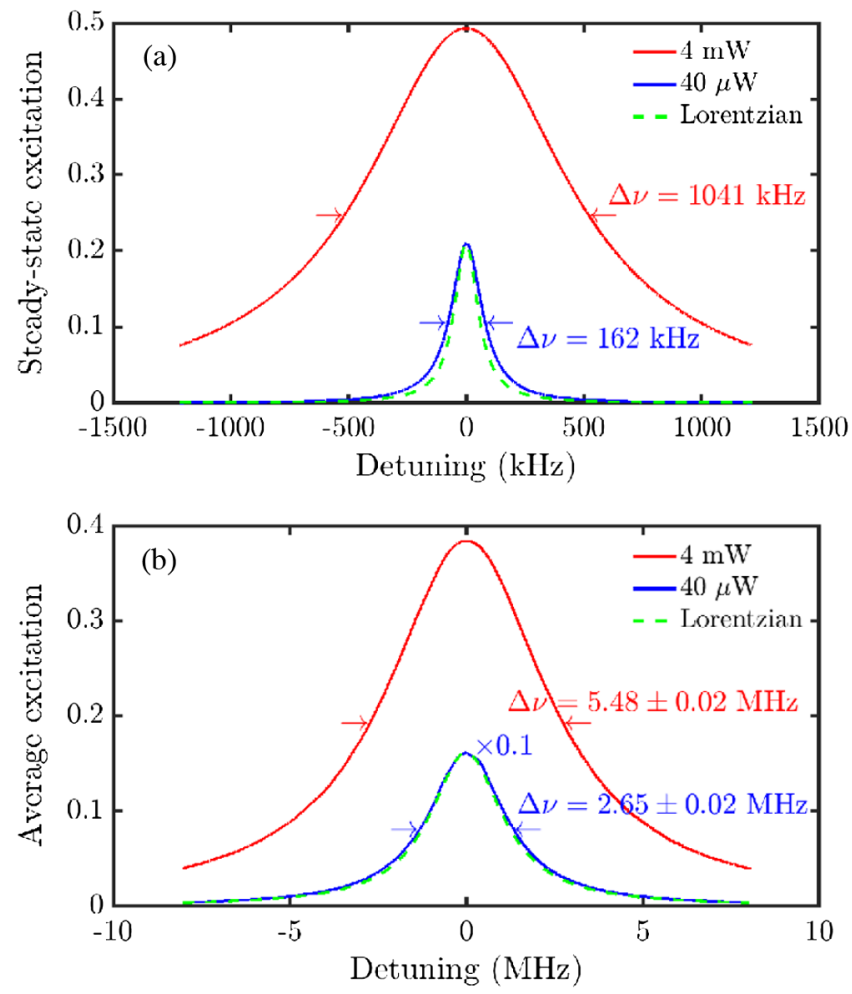

FIG. 3. Excitations vs detuning. (a) Steady-state excitations for comb 1 with $\tau_{c}=1 \mathrm{~s}$ [19]; (b) 1.3-ms-averaged excitations for comb 2 with $\tau_{c}=64$ ns [21]. For both cases, red and blue lines refer to $4-\mathrm{mW}$ and $40-\mu \mathrm{W}$ comb powers, respectively. The green dashed lines represent (a) the Lorentzian line shape of the 122-kHz-wide ${ }^{3} P_{1} \rightarrow{ }^{1} S_{0}$ ionic transition and (b) the 2.50-MHz-wide comb tooth.
For comb 2, whose $2.50-\mathrm{MHz}$ tooth width is 20 -fold broader than the natural linewidth, its excitation spectra depicted in Fig. 3(b) reveal the coherence properties of the comb itself. First, the spectrum induced by the 4-mW comb overestimates the tooth width by a factor of 2.2 due to power broadening, and predicts a relatively shorter coherence time of $29 \mathrm{~ns}$. However, with a $40-\mu \mathrm{W}$ power, one obtains the line shape of the comb tooth with a FWHM of $2.65 \pm 0.02 \mathrm{MHz}$ (the $0.02-\mathrm{MHz}$ uncertainty is obtained from 100 realizations of the spectra), thus providing a good determination of the 64-ns coherence time with a $6 \%$ deviation. Therefore, the temporal coherence of FCs can be verified on a timescale of several $\mu \mathrm{s}$, which is orders of magnitude longer than in previous experiments $[12,13,18,21]$. Furthermore, even for a comb coherence time as short as $64 \mathrm{~ns}$, Fig. 3(b) shows that DFCS of the ions could still improve the accuracy of the transition energy by five orders of magnitude.

The verification of the 1-s-long coherence time of comb 1 would require tuning $\omega_{0}$ to the extremely narrow, $30-\mathrm{mHz},{ }^{3} P_{2} \rightarrow{ }^{1} S_{0}$ forbidden transition around $14.331 \mathrm{eV}$. The effective Rabi frequencies of 35.6 and $356 \mathrm{~Hz}$ for the EUV comb powers of $40 \mu \mathrm{W}$ and $4 \mathrm{~mW}$, respectively, would result in hundreds to thousands of Rabi cycles before the system evolves into a dynamical steady state, thus enabling full quantum control of the corresponding ionic states. The simulated FWHMs of the spectra, however, show widths of $132 \mathrm{~Hz}$ and $1.25 \mathrm{kHz}$ for comb powers of $40 \mu \mathrm{W}$ and $4 \mathrm{~mW}$, respectively, due to power broadening. Though they are more than three orders of magnitude larger than the $30-\mathrm{mHz}$ natural linewidth and the $160-\mathrm{mHz}$ comb tooth width, they still represent an improvement in the accuracy of the ${ }^{3} P_{2} \rightarrow{ }^{1} S_{0}$ transition energy of $\mathrm{Ar}^{6+}$ to the level of $\delta \omega / \omega=10^{-14}$, and set up the lower bound of the EUV comb coherence time to the range of milliseconds. Nevertheless, one can eliminate the power broadening to obtain a more accurate determination of the coherence time (on the $10 \%$ level in our current example, limited by the finite lifetime of the ${ }^{3} P_{2}$ state) and of the transition energy by employing lower powers.

RFCS scheme.-Power broadening can be eliminated by implementing RFCS, where the ion is excited by two pulses separated from each other by $t_{n}=n T_{r}$ ( $n$ is the number of repetition cycles between the two pulses). When $t_{n} \ll \tau$, the total excitations by such a pulse-pair can be calculated as $[46,66]$

$$
\rho_{\mathrm{ee}}\left(t_{n}\right)=\frac{|\mu|^{2}}{2}\left(\mathcal{E}_{\mathrm{eff}} T_{r}\right)^{2}\left\{1+\cos \left[\Delta t_{n}+\phi\left(t_{n}\right)\right]\right\} .
$$

While the first term in the curly bracket of Eq. (7) describes the excitations resulting from two independent pulses (each contributes 1/2), the cosine term represents the Ramsey interference between the two pulses, thus generates Ramsey fringes and determines the transition 
frequency $[14,43,44,46]$. The derivation of Eq. (7) and the discussion of multipulse interferences can be found in the Supplemental Material [67]. Due to CEP dephasing, when $t_{n}$ becomes larger than $\tau_{c}$, there is no deterministic and reproducible phase relation between the two pulses. Therefore, the averaged excitation reduces to [22]

$$
\left\langle\rho_{\mathrm{ee}}\left(t_{n}\right)\right\rangle=\frac{|\mu|^{2}}{2}\left(\mathcal{E}_{\mathrm{eff}} T_{r}\right)^{2}\left[1+\cos \left(\Delta t_{n}\right) e^{-\frac{1}{2} \sigma^{2} t_{n}}\right] .
$$

The exponentially decaying term determines the coherence time of the applied pulse train. Since the field strength appears in Eq. (8) as a prefactor, power broadening is eliminated in this case [46]. Therefore, RFCS of $\mathrm{Ar}^{6+}$ ions can accurately measure the coherence time of the FC and, when the temporal coherence of comb 1 with $\tau_{c}=1 \mathrm{~s}$ is verified, infer the corresponding transition frequency in $\operatorname{Ar}^{6+}$ with $\delta \omega / \omega \sim 10^{-17}$.

Conclusions. - We show that the implementation of the direct and Ramsey frequency comb spectroscopy of highly charged Mg-like ions can allow the determination of the coherence time of EUV FCs at timescales of several seconds, up to seven orders of magnitude longer than in previous experiments, and improve the high-precision spectroscopy of EUV transitions by 12 orders of magnitude to the $\delta \omega / \omega \sim 10^{-17}$ level. An experimental demonstration of these presented results will open the door to quantum control [15] of highly charged ions with EUV light sources and enable applications of EUV transitions in the search for physics beyond the standard model such as the variation of the fine-structure constant and the potential existence of a fifth force [68].

*chunhai.lyu@mpi-hd.mpg.de

${ }^{\dagger}$ Present address: Department of Chemistry and Department of Physics and Astronomy, University of California, Irvine, California 92697-2025, USA.

[1] S. T. Cundiff and J. Ye, Rev. Mod. Phys. 75, 325 (2003).

[2] J. Ye and S. T. Cundiff, Femtosecond Optical Frequency Comb: Principle, Operation and Applications (Springer Science \& Business Media, New York, 2005).

[3] T. R. Schibli, I. Hartl, D. C. Yost, M. J. Martin, A. Marcinkevičius, M. E. Fermann, and J. Ye, Nat. Photonics 2, 355 (2008).

[4] C. Benko, A. Ruehl, M. J. Martin, K. S. E. Eikema, M. E. Fermann, I. Hartl, and J. Ye, Opt. Lett. 37, 2196 (2012).

[5] N. Picqué and T. W. Hänsch, Nat. Photonics 13, 146 (2019).

[6] T. Fortier and E. Baumann, Commun. Phys. 2, 1 (2019).

[7] C.-H. Li, A. J. Benedick, P. Fendel, A. G. Glenday, F. X. Kärtner, D. F. Phillips, D. Sasselov, A. Szentgyorgyi, and R. L. Walsworth, Nature (London) 452, 610 (2008).

[8] T. Steinmetz, T. Wilken, C. Araujo-Hauck, R. Holzwarth, T. W. Hänsch, L. Pasquini, A. Manescau, S. D’Odorico, M. T. Murphy, T. Kentischer, W. Schmidt, and T. Udem, Science 321, 1335 (2008).
[9] A. D. Ludlow, M. M. Boyd, J. Ye, E. Peik, and P. O. Schmidt, Rev. Mod. Phys. 87, 637 (2015).

[10] A. K. Mills, T. J. Hammond, M. H. C. Lam, and D. J. Jones, J. Phys. B 45, 142001 (2012).

[11] C. Gohle, T. Udem, M. Herrmann, J. Rauschenberger, R. Holzwarth, H. A. Schuessler, F. Krausz, and T. W. Hänsch, Nature (London) 436, 234 (2005).

[12] R. J. Jones, K. D. Moll, M. J. Thorpe, and J. Ye, Phys. Rev. Lett. 94, 193201 (2005).

[13] A. Cingöz, D. C. Yost, T. K. Allison, A. Ruehl, M. E. Fermann, I. Hartl, and J. Ye, Nature (London) 482, 68 (2012)

[14] L. S. Dreissen, C. Roth, E. L. Gründeman, J. J. Krauth, M. Favier, and K. S. E. Eikema, Phys. Rev. Lett. 123, 143001 (2019).

[15] B. W. Adams, C. Buth, S. M. Cavaletto, J. Evers, Z. Harman, C. H. Keitel, A. Pálffy, A. Picón, R. Röhlsberger, Y. Rostovtsev et al., J. Mod. Opt. 60, 2 (2013).

[16] S. M. Cavaletto, Z. Harman, C. Ott, C. Buth, T. Pfeifer, and C. H. Keitel, Nat. Photonics 8, 520 (2014).

[17] B. Seiferle, L. von der Wense, P. V. Bilous, I. Amersdorffer, C. Lemell, F. Libisch, S. Stellmer, T. Schumm, C. E. Düllmann, A. Pálffy, and P. G. Thirolf, Nature (London) 573, 243 (2019).

[18] D. C. Yost, T. R. Schibli, J. Ye, J. L. Tate, J. Hostetter, M. B. Gaarde, and K. J. Schafer, Nat. Phys. 5, 815 (2009).

[19] C. Benko, T. K. Allison, A. Cingöz, L. Hua, F. Labaye, D. C. Yost, and J. Ye, Nat. Photonics 8, 530 (2014).

[20] D. G. Matei, T. Legero, S. Häfner, C. Grebing, R. Weyrich, W. Zhang, L. Sonderhouse, J. M. Robinson, J. Ye, F. Riehle et al., Phys. Rev. Lett. 118, 263202 (2017).

[21] C. Corsi, I. Liontos, M. Bellini, S. Cavalieri, P. Cancio Pastor, M. Siciliani de Cumis, and R. Eramo, Phys. Rev. Lett. 118, 143201 (2017).

[22] R. Eramo, P. Cancio Pastor, and S. Cavalieri, Phys. Rev. A 97, 033842 (2018).

[23] P. Micke, S. Kühn, L. Buchauer, J. R. Harries, T. M. Bücking, K. Blaum, A. Cieluch, A. Egl, D. Hollain, S. Kraemer et al., Rev. Sci. Instrum. 89, 063109 (2018).

[24] M. Schwarz, O. O. Versolato, A. Windberger, F. R. Brunner, T. Ballance, S. N. Eberle, J. Ullrich, P. O. Schmidt, A. K. Hansen, A. D. Gingell, M. Drewsen, and J. R. Crespo López-Urrutia, Rev. Sci. Instrum. 83, 083115 (2012).

[25] T. Leopold, S. A. King, P. Micke, A. Bautista-Salvador, J. C. Heip, C. Ospelkaus, J. R. Crespo López-Urrutia, and P. O. Schmidt, Rev. Sci. Instrum. 90, 073201 (2019).

[26] P. Micke, T. Leopold, S. A. King, E. Benkler, L. J. Spieß, L. Schmöger, M. Schwarz, J. R. Crespo López-Urrutia, and P. O. Schmidt, Nature (London) 578, 60 (2020).

[27] J. Nauta, A. Borodin, H. B. Ledwa, J. Stark, M. Schwarz, L. Schmöger, P. Micke, J. R. Crespo López-Urrutia, and T. Pfeifer, Nucl. Instrum. Methods Phys. Res., Sect. B 408, 285 (2017).

[28] J. Nauta, J.-H. Oelmann, A. Ackermann, P. Knauer, R. Pappenberger, A. Borodin, I. S. Muhammad, H. Ledwa, T. Pfeifer, and J. R. Crespo López-Urrutia, Opt. Lett. 45, 2156 (2020).

[29] J. R. Crespo López-Urrutia, J. Phys. Conf. Ser. 723, 012052 (2016). 
[30] M. G. Kozlov, M. S. Safronova, J. R. Crespo López-Urrutia, and P. O. Schmidt, Rev. Mod. Phys. 90, 045005 (2018).

[31] J. C. Berengut, V. A. Dzuba, and V. V. Flambaum, Phys. Rev. Lett. 105, 120801 (2010).

[32] J. C. Berengut, V. A. Dzuba, V. V. Flambaum, and A. Ong, Phys. Rev. Lett. 109, 070802 (2012).

[33] H. Bekker, A. Borschevsky, Z. Harman, C. H. Keitel, T. Pfeifer, P. O. Schmidt, J. R. Crespo López-Urrutia, and J. C. Berengut, Nat. Commun. 10, 5651 (2019).

[34] A. Windberger, J. R. Crespo López-Urrutia, H. Bekker, N. S. Oreshkina, J. C. Berengut, V. Bock et al., Phys. Rev. Lett. 114, 150801 (2015).

[35] J. C. Berengut, D. Budker, C. Delaunay, V. V. Flambaum, C. Frugiuele, E. Fuchs, C. Grojean, R. Harnik, R. Ozeri, G. Perez, and Y. Soreq, Phys. Rev. Lett. 120, 091801 (2018).

[36] V. Debierre, C. H. Keitel, and Z. Harman, Phys. Lett. B 807, 135527 (2020).

[37] S. Gustafsson, P. Jönsson, C. F. Fischer, and I. P. Grant, Astron. Astrophys. 597, A76 (2017).

[38] C. F. Fischer, G. Gaigalas, P. Jönsson, and J. Bieroń, Comput. Phys. Commun. 237, 184 (2019).

[39] A. Kramida, Y. Ralchenko, and J. Reader (NIST ASD Team), NIST Atomic Spectra Database (Version 5.7.1), https://physics.nist.gov/asd (2019).

[40] S. Cavalieri, R. Eramo, M. Materazzi, C. Corsi, and M. Bellini, Phys. Rev. Lett. 89, 133002 (2002).

[41] R. Eramo, S. Cavalieri, C. Corsi, I. Liontos, and M. Bellini, Phys. Rev. Lett. 106, 213003 (2011).

[42] D. Z. Kandula, C. Gohle, T. J. Pinkert, W. Ubachs, and K. S. E. Eikema, Phys. Rev. Lett. 105, 063001 (2010).

[43] S. Witte, R. T. Zinkstok, W. Ubachs, W. Hogervorst, and K. S. E. Eikema, Science 307, 400 (2005).

[44] R. K. Altmann, L. S. Dreissen, E. J. Salumbides, W. Ubachs, and K. S. E. Eikema, Phys. Rev. Lett. 120, 043204 (2018).

[45] D. Felinto and C. E. E. López, Phys. Rev. A 80, 013419 (2009).

[46] J. Morgenweg, I. Barmes, and K. S. E. Eikema, Nat. Phys. 10, 30 (2014).

[47] A. G. Trigueiros, A. J. Mania, M. Gallardo, and J. G. Reyna Almandos, J. Opt. Soc. Am. B 14, 2463 (1997).

[48] M. Endo, T. D. Shoji, and T. R. Schibli, IEEE J. Sel. Top. Quantum Electron. 24, 1 (2018).

[49] A. Bartels, C. W. Oates, L. Hollberg, and S. A. Diddams, Opt. Lett. 29, 1081 (2004).
[50] A. Liehl, P. Sulzer, D. Fehrenbacher, T. Rybka, D. V. Seletskiy, and A. Leitenstorfer, Phys. Rev. Lett. 122, 203902 (2019).

[51] R. J. Temkin, J. Opt. Soc. Am. B 10, 830 (1993).

[52] R. W. Ziolkowski, J. M. Arnold, and D. M. Gogny, Phys. Rev. A 52, 3082 (1995).

[53] N. V. Vitanov and P. L. Knight, Phys. Rev. A 52, 2245 (1995).

[54] M. Scully and S. Zubairy, Quantum Optics (Cambridge University Press, Cambridge, England, 1996).

[55] G. Porat, C. M. Heyl, S. B. Schoun, C. Benko, N. Dörre, K. L. Corwin, and J. Ye, Nat. Photonics 12, 387 (2018).

[56] D. Felinto, C. A. C. Bosco, L. H. Acioli, and S. S. Vianna, Phys. Rev. A 64, 063413 (2001).

[57] D. Felinto, C. A. C. Bosco, L. H. Acioli, and S. S. Vianna, Opt. Commun. 215, 69 (2003).

[58] A. Marian, M. C. Stowe, J. R. Lawall, D. Felinto, and J. Ye, Science 306, 2063 (2004).

[59] D. Aumiler, T. Ban, and G. Pichler, Phys. Rev. A 79, 063403 (2009).

[60] L. Allen and J. H. Eberly, Optical Resonance and Two-Level Atoms (Wiley, New York, 1975).

[61] M. P. Moreno and S. S. Vianna, Opt. Commun. 313, 113 (2014).

[62] V. I. Yudin, A. V. Taichenachev, and M. Y. Basalaev, Phys. Rev. A 93, 013820 (2016).

[63] S. M. Cavaletto, Ph.D thesis, Unviersity of Heidelberg, 2013, https://doi.org/10.11588/heidok.00015982.

[64] M. A. Newbold and G. J. Salamo, Phys. Rev. A 22, 2098 (1980).

[65] S. M. Cavaletto, Z. Harman, C. Buth, and C. H. Keitel, Phys. Rev. A 88, 063402 (2013).

[66] The phase $\phi\left(t_{n}\right)$ in Eq. (7) is the phase of the pulse at $t$ after subtracting the running-wave phase $\omega_{0} t_{n}$, see also Eq. (1). As an equivalent form, the cosine term in Eq. (7) can also be written as $\cos \left[2 \pi f_{\text {tr }} t_{n}-\varphi\left(t_{n}\right)\right]$. Here, $f_{\text {tr }}$ represents the ionic transition frequency, while $\varphi\left(t_{n}\right)=2 \pi f_{0} t_{n}+\phi\left(t_{n}\right)$ $\left(f_{0}=\omega_{0} / 2 \pi\right)$ is the global phase difference between the two pulses that also includes the running-wave phase $\omega_{0} t_{n}=2 \pi f_{0} t_{n}$.

[67] See the Supplemental Material at http://link.aps.org/ supplemental/10.1103/PhysRevLett.125.093201 for the detailed derivations and discussions of the excitations induced by multiple pulses in the case of weak excitations.

[68] M. S. Safronova, D. Budker, D. DeMille, Derek F. Jackson Kimball, A. Derevianko, and C. W. Clark, Rev. Mod. Phys. 90, 025008 (2018). 